\title{
Hybrid Polymer Nanoassemblies: Polymer Nanosheets Organized with Metal Nanoparticle Arrays for Surface Plasmon Photonics
}

\author{
Masaya Mitsuishi, ${ }^{\dagger}$ Miki IshifuJI, Hiroshi Endo, Hiroyuki TANAKA, and Tokuji MiYashita \\ Institute of Multidisciplinary Research for Advanced Materials (IMRAM), Tohoku University, \\ 2-1-1 Katahira, Aoba-ku, Sendai 980-8577, Japan
}

(Received February 8, 2007; Accepted February 23, 2007; Published March 30, 2007)

\begin{abstract}
The paper describes fabrication of hybrid polymer nanoassemblies possessing polymer nanosheets and metal nanoparticle arrays. Based on a bottom-up approach with ultrathin polymer Langmuir-Blodgett films (polymer nanosheets) and metal nanoparticles, we constructed hybrid nanoassemblies for opto/electric nanodevice applications: nanoscale positioning of metal nanoparticles, free-standing hybrid polymer nanosheets, hybrid polymer nanoassemblies for luminescence sensor application, and enhancement of nonlinear optical (NLO) efficiency via surface plasmon coupling. Assembling nanomaterials such as nanoparticles and organic functional molecules through a bottom-up approach makes it possible to strengthen free-standing nanofilm stability, enhance luminescence of luminescent molecules, and enhance second harmonic light from NLO molecules under the influence of localized surface plasmons. The high potential of hybrid polymer nanoassemblies for precise nanoscale design is also demonstrated.
\end{abstract} [doi:10.1295/polymj.PJ2006235]

KEY WORDS Polymer / LB Film / Metal Nanoparticle / Surface Plasmon / Hybrid /

Design of nanostructures using polymer materials has been widely explored and has now become prevalent. Bottom-up approaches such as layer-by-layer (LbL) adsorption, ${ }^{1}$ surface-initiated polymerization exemplified by atom transfer radical polymerization (ATRP) ${ }^{2}$ and Langmuir-Blodgett (LB) technique ${ }^{3-7}$ make it possible to develop myriad nanoarchitectures on solid substrates. These approaches and their combinations offer a promising route to polymer nanoarchitectures for a wide variety of functional materials and surfaces. Using these nanostructures, for instance, the surface properties can be controlled uniformly, even on a nanoscale. These compelling qualities of ultrathin polymer films have been expanded to broad scientific fields. It is important to intensify the interdisciplinary interactions, for example, between polymer materials and inorganic nanoparticles in basic and application-oriented work.

We have investigated functionalization of polymer LB films consisting of poly(alkylacrylamide)s. Poly( $N$-dodecylacrylamide) (pDDA) proved to be excellent for LB assembly; the material provides a highly oriented and densely packed monolayer at the airwater interface. ${ }^{8,9}$ Varying the side chain length, the film thickness is tuned at a nanometer scale $(1-2 \mathrm{~nm}$ in length). Widely various functional molecules were incorporated and distributed uniformly in a two-dimensional (2D) field. The key factor is the existence of a $2 \mathrm{D}$ hydrogen-bonding network between polymer backbones, which enhances the monolayer stability at the air-water interface, leading to quantitative deposition onto solid substrates via vertical dipping. We recently designated polymer LB films consisting of alkyl acrylamide polymer as polymer nanosheets. Using these advantages, directional (vectorial) electron transfer between several redox components was achieved in multilayered polymer nanosheets, ${ }^{10,11} \mathrm{ex}-$ tending to logic gate operations. ${ }^{12,13}$ We have demonstrated photochemically patterned polymer nanosheets for three-dimensional (3D) nanoarchitecture. ${ }^{14-17}$ Compared to low-molecular-weight fatty acids and other polymer LB films, the pDDA nanosheets give simplicity and versatility in preparation and usage. In fact, preparation of pDDA monolayers requires no addition of ions to the water subphase to stabilize the monolayer formation. The pDDA monolayers were deposited onto numerous substrates, such as quartz, glass, ITO glass, ${ }^{10,11}$ metals, ${ }^{18,19}$ plastic fiber, and spin-coated films.

Recently, metal nanoparticles have received much attention ${ }^{20}$ because they show fascinating features such as quantum size effect, catalytic activity, and localized surface plasmon. ${ }^{21-23} \mathrm{We}$ investigated preparation of metal nanoparticle arrays with polymer nanosheets through electrostatic interaction. Polymer nanosheets, which consist of cationic comonomer and $\mathrm{N}$-dodecylacrylamide capable of forming LB films, serve as a template for metal nanoparticle im-

${ }^{\dagger}$ To whom correspondence should be addressed (Tel/Fax: +81-22-217-5638, E-mail: masaya@tagen.tohoku.ac.jp). 


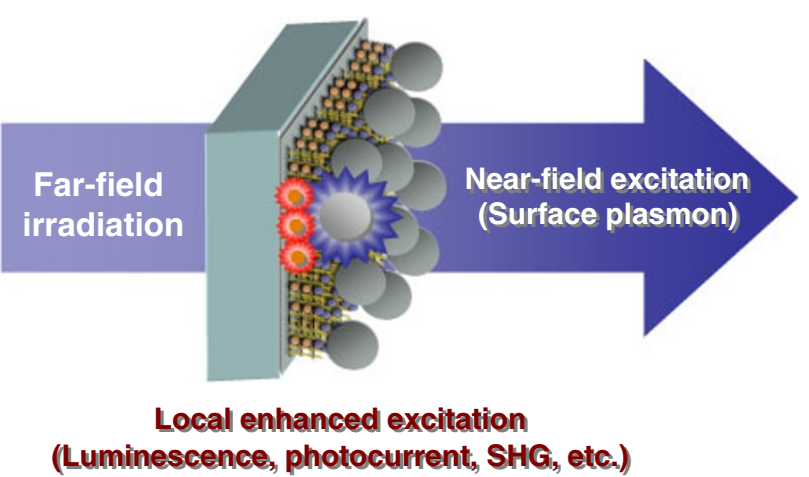

Figure 1. Schematic illustration of hybrid polymer nanoassemblies.

mobilization. We assembled functional polymer nanosheets with metal nanoparticle monolayers to utilize a localized surface plasmon as an excitation source (Figure 1). The hybrid polymer nanoassemblies show strong extinction bands in the visible light wavelength because of the localized surface plasmon resonance of metal nanoparticle arrays. They are expected to be available for opto/electronic device application based on their surface plasmon photonics. In this paper, we specifically address recent topics of hybrid polymer nanoassemblies via polymer nanosheets. We describe a) fabrication of hybrid polymer nanoassemblies via a bottom-up approach based on the LB technique, b) robust and flexible free-standing polymer nanosheets organized with photo-cross-linkable polymer nanosheets and gold nanoparticles, c) spectroscopic characterization of hybrid polymer nanoassemblies for luminescence sensor applications, d) enhanced second harmonic generation from hybrid polymer nanoassemblies, and e) hybrid polymer nanoassemblies for precise nanoscale design. The potential applications and advantages of the hybrid polymer nanoassemblies for surface plasmon photonics are also discussed.

\section{FABRICATION OF HYBRID POLYMER NANOASSEMBLIES}

Numerous reports have described new architectures assembled with metal nanoparticles and polymer materials. ${ }^{24}$ As described in a previous section, LbL assembly, LB technique, and self-assembled monolayer method have emerged as popular and versatile approaches. Our strategy for hybrid polymer nanoassemblies is very straightforward: ${ }^{25}$ cationic polymer nanosheets are transferred onto solid substrates. Subsequently, the substrates are immersed in metal nanoparticle aqueous solution, then rinsed in distilled water and dried with nitrogen gas (Figure 2). For that process, amphiphilic copolymers containing a pyridyl (VPy) or amino (DADOO) group were synthesized through radical copolymerization ( $\mathrm{p}(\mathrm{DDA} / \mathrm{VPy})$ and $\mathrm{p}$ (DDA/DADOO), Figure 2). ${ }^{14,16}$ These materials have high LB-film-formation capability and provide densely packed and highly oriented polymer LB films with vertical dipping method. The polymer nanosheets serve as good templates for metal nanoparticle ordering. Metal nanoparticles were synthesized through citrate reduction method. ${ }^{20 a, 26}$ Using this method, monodispersed metal nanoparticles were obtained, the surfaces of which were covered by negatively charged citrate anions at normal $\mathrm{pH}$ conditions. Through immersion of the substrates coated with the polymer nanosheets in metal nanoparticle aqueous solution, the metal nanoparticles became immobilized uniformly on the cationic polymer nanosheets through electrostatic interaction. On the polymer nanosheets, well-separated metal nanoparticle monolayer formation was achieved, probably because of electrostatic repulsion between negatively charged metal nanoparticles (Figure 3). These nanostructures are strongly stable over one year, although particle aging resulting
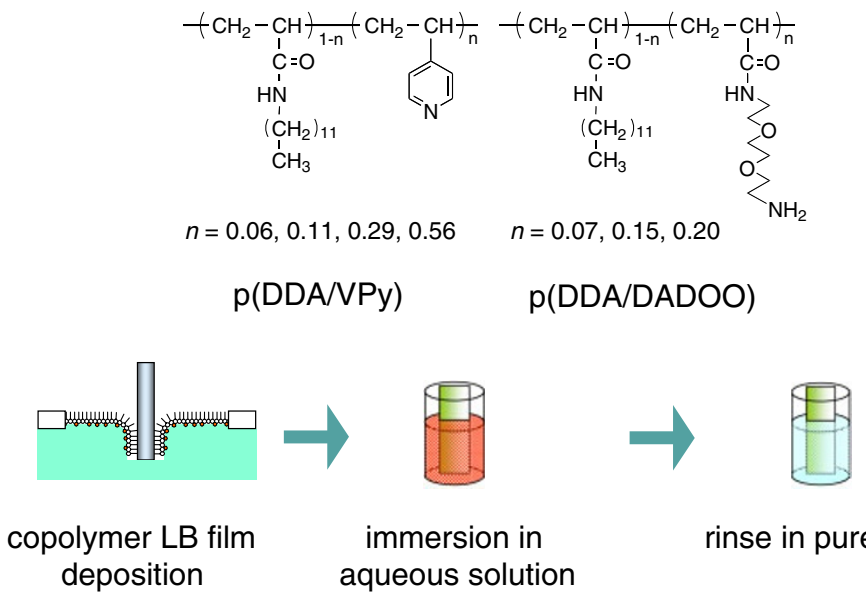

rinse in pure water

Figure 2. Chemical structure of cationic polymer nanosheets and schematic illustration of the preparation process of hybrid polymer nanoassemblies. 


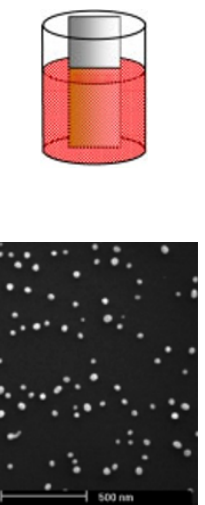

$30 \mathrm{~min}$

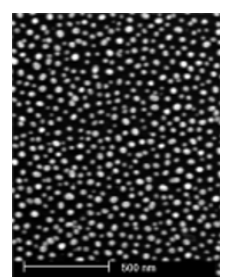

$8 \mathrm{~h}$

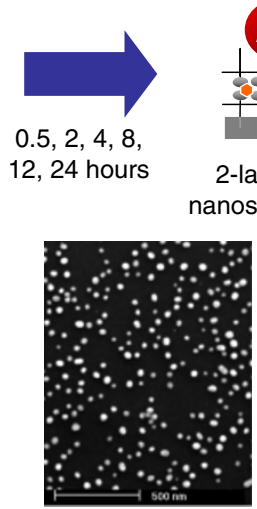

$2 \mathrm{~h}$

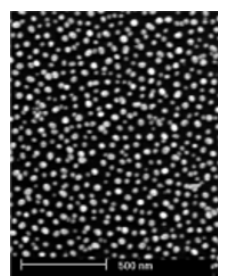

$12 \mathrm{~h}$
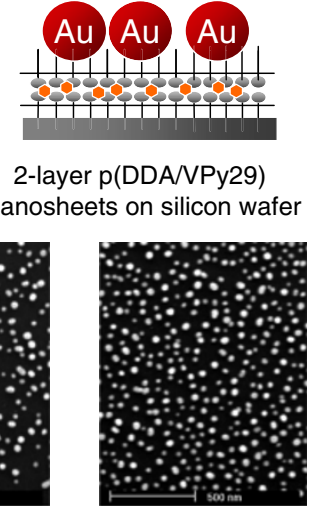

$4 \mathrm{~h}$

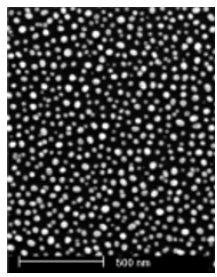

$24 \mathrm{~h}$
Figure 3. SEM images of gold nanoparticle arrays immobilized on two-layer $\mathrm{p}(\mathrm{DDA} / \mathrm{VPy})$ nanosheets with different VPy contents.

from oxidation or contamination might affect spectroscopic properties, which reflects specific interaction between metal nanoparticles and cationic polymer nanosheets with multiple linkages. Control of lateral metal nanoparticle ordering is also possible by varying the molar contents of VPy or DADOO in the polymer nanosheets.

The pDDA nanosheets were transferred on $\mathrm{p}(\mathrm{DDA} /$ VPy29) (VPy contents $=29(\mathrm{~mol} \%))$ nanosheets, as a function of the number of layers, to examine the effect of cationic polymer nanosheets on metal nanoparticle immobilization. Figure 4 shows that the amount of immobilized gold nanoparticles depends strongly on the spacer length. The amount decreased as the number of deposited pDDA spacer layers increased. Eventually no adsorption of gold nanoparticles (apart from those immobilized through nonspecific physisorption) was observed over five-layer pDDA nanosheets. The findings imply that a long-range electrostatic interaction exists between gold nanoparticle and p(DDA/ VPy) nanosheets and that this interaction is a predominant driving force for gold nanoparticle immobilization. As for the effect of cationic polymer nanosheets, the surface charge density is also controllable by changing the number of deposited cationic polymer nanosheets; the amount of immobilized gold nanoparticles was saturated above two-layer cationic polymer nanosheets.

\section{FREE-STANDING ULTRATHIN HYBRID POLYMER NANOASSEMBLIES}

Free-standing membranes, which are decoupled from supporting substrates, are of great interest for use in micromechanical devices, sensors, and actuators. The term free-standing means that the films require no full contact with a solid substrate to sustain their shape and properties in air or liquid. In other words, films that are partially supported by solid substrates (such as a copper grid or those with one end anchored) are included in this category. Their flexibility is distinctly advantageous for various applications; their shape and dimensions can be changed. In general, preparation of free-standing ultrathin polymer films requires two steps: film growth and removal on a substrate. Many studies have been undertaken to develop and establish fabrication procedures using a bottomup approach. Several different approaches based on LbL assembly and LB techniques have reportedly
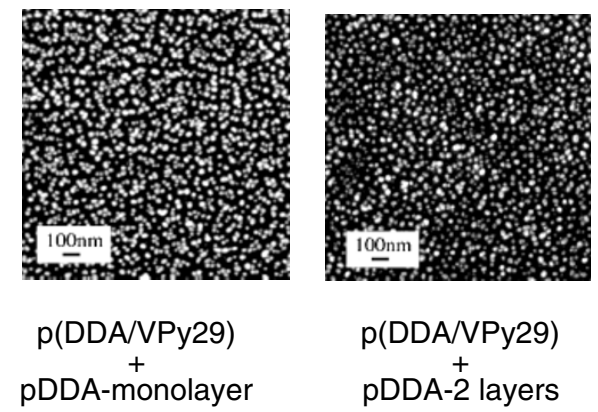

pDDA-monolayer

$$
\begin{gathered}
\text { p(DDA/VPy29) } \\
++ \\
\text { pDDA-2 layers }
\end{gathered}
$$
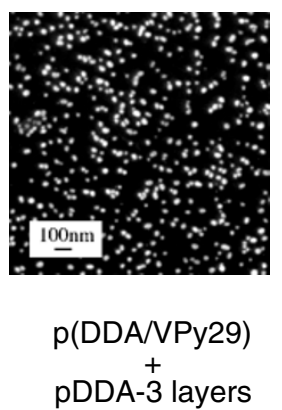

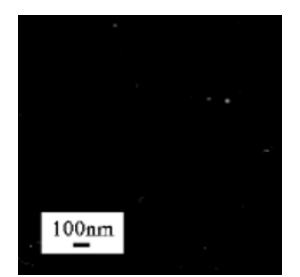

$\mathrm{p}(\mathrm{DDA} / \mathrm{VPy} 29)$

pDDA- $\stackrel{+}{5}$ layers

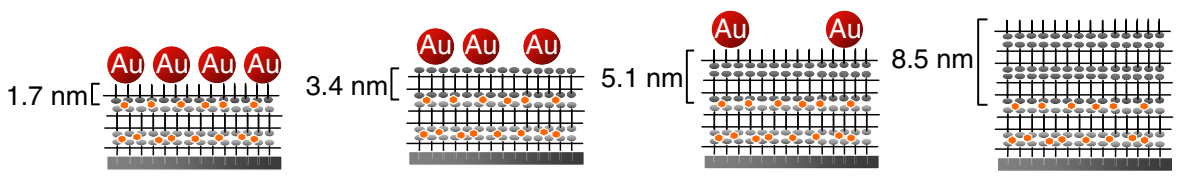

Figure 4. SEM images of gold nanoparticle arrays immobilized on p(DDA/VPy) nanosheets as a function of the number of pDDA outermost layers. 


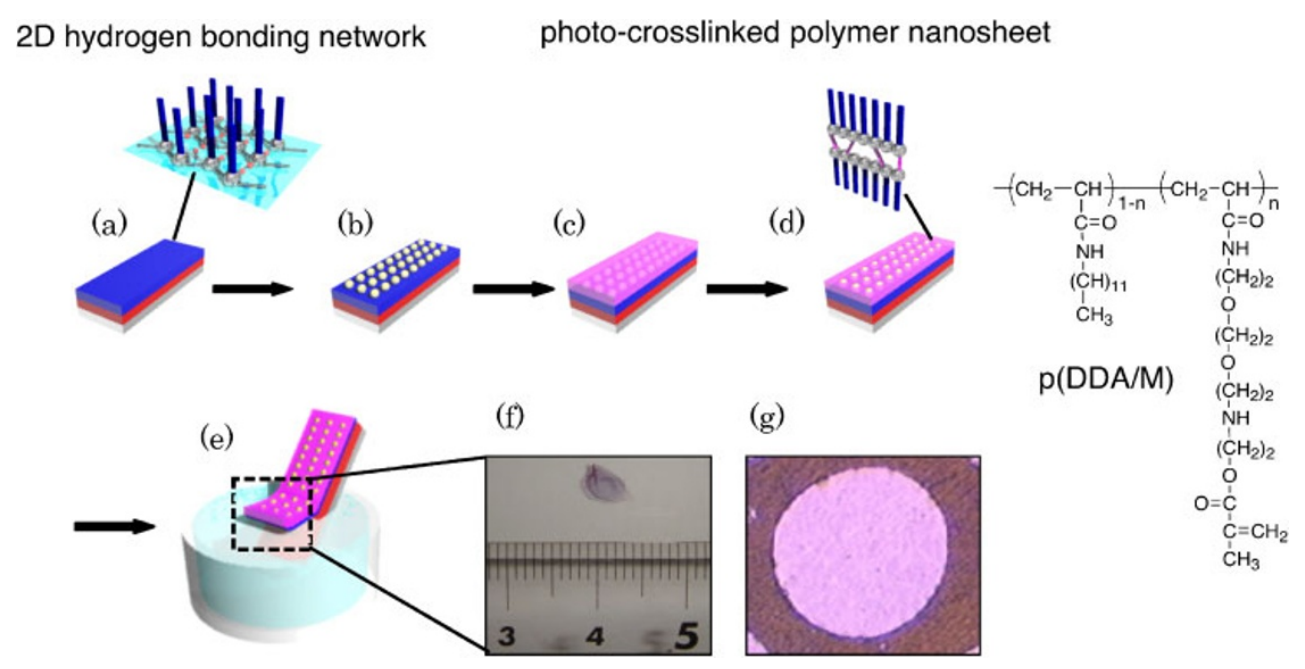

Figure 5. Schematic illustration of free-standing hybrid polymer nanoassemblies. Reprinted with permission from ref 35 . Copyright 2006, American Chemical Society.

yielded robust and tough free-standing films..$^{27-34}$ Various inorganic nanomaterials such as magnetic materials, semiconductors, metal nanoparticles, and carbon nanotubes have been incorporated into free-standing films. Moreover, chemical or physical cross-linking has been found to strengthen and stabilize free-standing films. Combining incorporation of metal nanoparticles and photo-cross-linking reactions into polymer nanosheets, we fabricated free-standing polymer nanofilms. ${ }^{35}$ The experimental outline of free-standing hybrid polymer nanosheets is shown schematically in Figure 5. Ten-layer pDDA nanosheets, sacrificed layers, were transferred to a silicon wafer, and twolayer $\mathrm{p}(\mathrm{DDA} / \mathrm{DADOO})$ nanosheets were transferred to immobilize gold nanoparticles. The surface was coated with photo-cross-linkable polymer nanosheets (p(DDA/M), see Figure 5). After photo-irradiation with a deep UV lamp, the substrate was immersed in chloroform. The ultrathin film was removed from the surface. Figure 5 shows that the holes $(85 \mu \mathrm{m}$ diameter) of the copper TEM grid were covered with the free-standing hybrid polymer nanosheets. It is worth noting that it was difficult to peel off the film while leaving the gold nanoparticle layer in this procedure. In other words, gold nanoparticles reinforce and stabilize the free-standing structure as nano-filler material. We prepared free-standing hybrid polymer nanosheets that were larger than $50 \mathrm{~mm}^{2}$ and which showed high durability of several months. Figure 6 shows AFM images of the resultant hybrid free-standing polymer nanosheets. The film thickness was determined as approximately $30 \mathrm{~nm}$, which is the same as the diameter of gold nanoparticles that were mixed into the film. Assuming that the $\mathrm{p}(\mathrm{DDA} / \mathrm{M})$ nanosheets take a fairly flat multilayer structure on the gold nanoparticle layer, the layer thickness is $2.0 \times 14=28$ $(\mathrm{nm})$. Considering the free-standing film thickness
$(30 \mathrm{~nm})$ and the surface coverage of gold nanoparticles (42\%), the volume of the $\mathrm{p}(\mathrm{DDA} / \mathrm{M})$ nanosheets is sufficient to occupy the gap between gold nanoparticles. Furthermore, the $\mathrm{p}(\mathrm{DDA} / \mathrm{M})$ nanosheets undergo a photo-cross-linking reaction during deep UV irradiation and might take thermal relaxation, keeping the gold nanoparticles separated from each other. Consequently, the free-standing hybrid polymer nanosheet has a uniform distribution of gold nanoparticles and a smooth surface (RMS $<5 \mathrm{~nm}$ ). These results indicate that gold nanoparticles make polymer nanosheets robust and easy to release from solid supports. As for sacrificing the layer, the peeling test as a function of the number of pDDA spacer layers showed that a critical length existed: above 10 layers ( $c a .17 \mathrm{~nm}$ thick). No detachment from the substrate was observed below six layers and mechanical oscillation was necessary for eight layers.

\section{SPECTROSCOPIC PROPERTIES OF METAL NANOPARTICLES ASSEMBLED WITH POLYMER NANOSHEETS}

Spectroscopic characterization of metal nanoparticles on a solid substrate sometimes meets difficulty in determination because metal nanoparticles tend to take an aggregate formation. In solution, the molar extinction coefficient of metal nanoparticles is determined quantitatively using Mie theory. ${ }^{36}$ A deep understanding of spectroscopic properties of metal nanoparticle will help us find new functionality that is related to photonic and electronic device application. We attempted to examine spectroscopic properties of metal nanoparticles by preparing uniformly distributed metal nanoparticle monolayers. We then carried out ESEM observation and UV-Vis extinction spectra measurements. Figure 7 shows an environmental scan- 

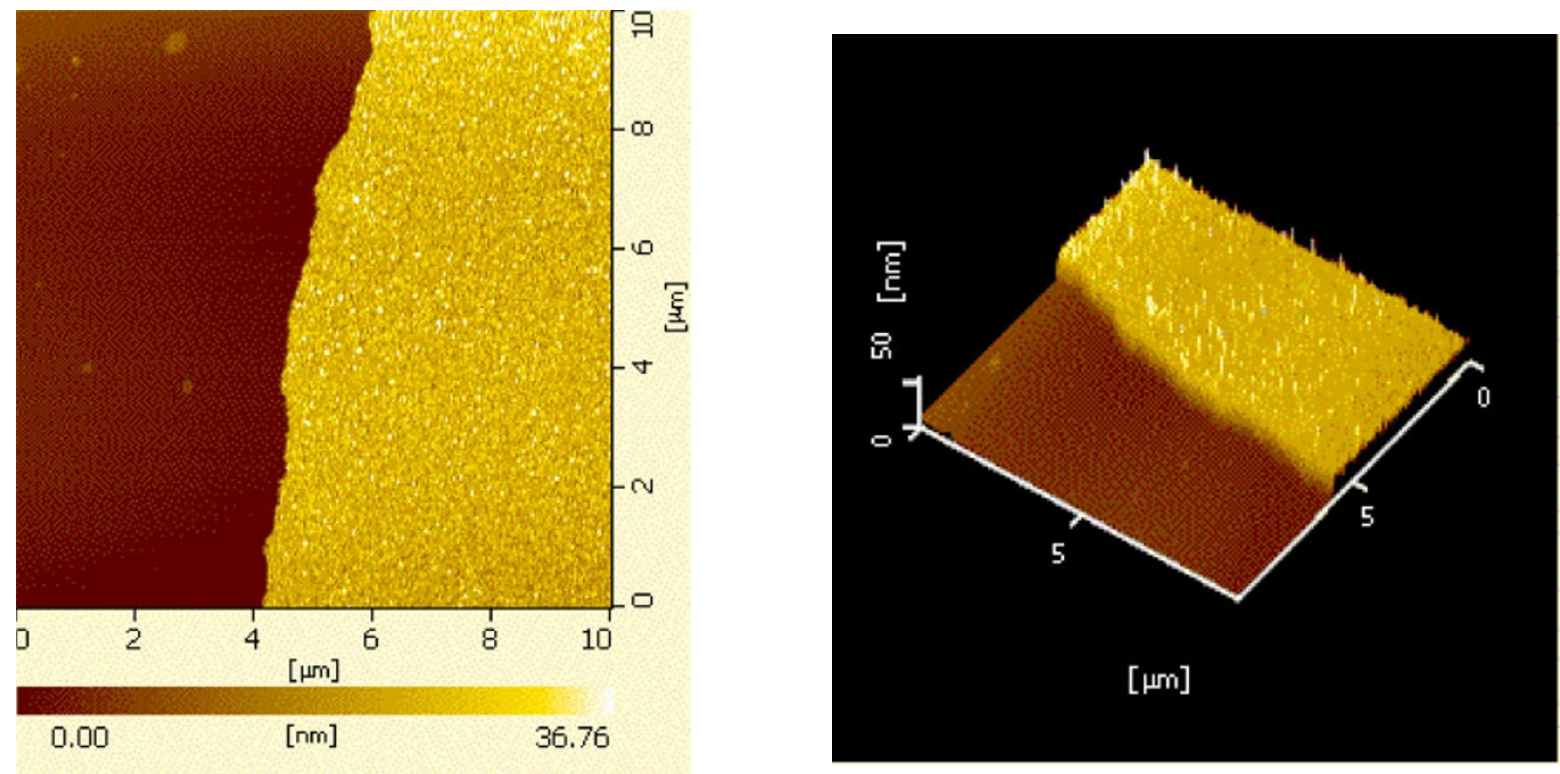

Figure 6. AFM images of free-standing hybrid polymer nanoassemblies supported by a silicon wafer.

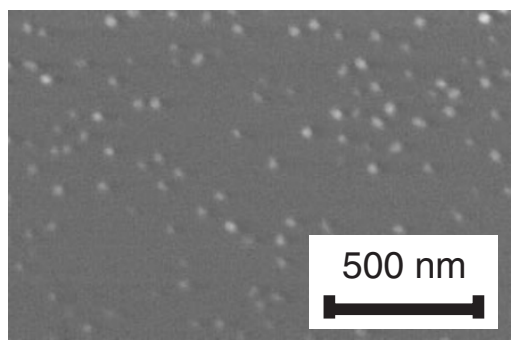

Figure 7. SEM image of hybrid polymer nanoassemblies on a quartz slide.

ning electron microscope (ESEM) image of gold nanoparticles immobilized on two-layer p(DDA/VPy29) nanosheets on a transparent glass substrate after $4 \mathrm{~h}$ immersion. In this condition, the gold nanoparticles take no notable aggregate formation on p(DDA/ VPy) nanosheets: almost all the gold nanoparticles are isolated from their neighbors. The UV-Vis extinction spectrum of the substrate exhibits a strong extinction band at $525 \mathrm{~nm}$ because of dipole-like surface plasmon resonance, i.e., collective oscillation of free electron gas in the gold nanoparticles coupled with incident light (Figure 8). The ESEM image shows that the surface density of the gold nanoparticle was determined as $51 \pm 3 / \mu \mathrm{m}^{2}$. We can determine the molar extinction coefficient of the gold nanoparticle on substrates, assuming that the reflection loss from the gold nanoparticle is negligible in UV-Vis extinction measurement: $7.1 \times 10^{12} \mathrm{M}^{-1} \mathrm{~cm}^{-1}$ for gold nanoparticles $(30 \mathrm{~nm} \phi)$. It is noteworthy that the molar extinction coefficient includes the contribution from both absorption and scattering caused by surface plasmon resonance. ${ }^{37}$ Considering the metal nanoparticle size, the contribution from absorption seems to be predominant

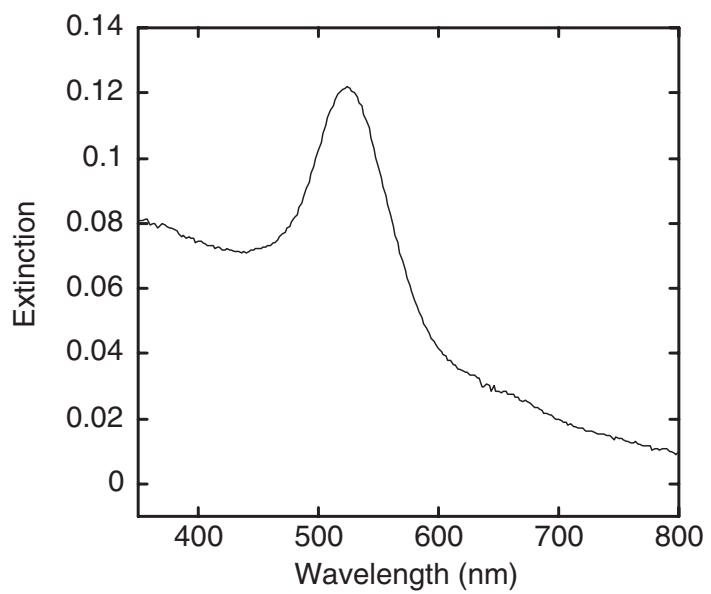

Figure 8. UV-Vis extinction spectrum of hybrid polymer nanoassemblies on a quartz slide.

for smaller gold nanoparticles $(30 \mathrm{~nm} \phi) .{ }^{38}$ Similarly, the molar extinction coefficient of silver nanoparticles ((averaged) $80 \mathrm{~nm} \phi$ ) was determined as roughly $9.5 \times$ $10^{12} \mathrm{M}^{-1} \mathrm{~cm}^{-1}$. These large molar extinction coefficient values ${ }^{39,40}$ imply that metal nanoparticles serve as an effective acceptor in photoexcited energy transfer processes. For gold and silver nanoparticles, the respective optical extinctions at $\lambda_{\max }$ of an aqueous metal nanoparticle solution for immobilization were $1.4(10 \mathrm{~mm}$ cell $)$ and 1.5 ( $2 \mathrm{~mm}$ cell). From these arguments, the concentration of metal nanoparticles in aqueous solution is inferred to be lower, resulting in a longer time for saturation of adsorption in a previous report. ${ }^{31}$

Ruthenium complexes are well known luminescent molecules. They have high quantum yield, long excited-state lifetime, and strong absorption in the bluegreen spectral region. Broad structureless emissions 
arise from metal-to-ligand charge-transfer (MLCT). The long microsecond lifetime comes from excited states containing a high degree of triplet character. The luminescence is quenched easily using molecular oxygen. Consequently, the material, along with platinum-porphyrin, is useful as a luminescence sensor for oxygen ${ }^{41-44} \mathrm{We}$ incorporated ruthenium complexes in polymer nanosheets through polyion complex meth$\mathrm{od}^{45}$ and examined the possibility of the luminescent polymer nanosheets for oxygen sensor applications. The luminescent polymer nanosheet provides smooth surface coating, and enables the surface oxygen concentration detection as the luminescence intensity changes. Faster optical response is expected using these ultrathin films. However, it seems to be difficult to gain sufficient luminescence intensity because of insufficiently numerous luminophores in the ultrathin film. We tried to enhance the luminescence from ruthenium complexes incorporated in polymer nanosheets using a localized surface plasmon electromagnetic field as an excitation source to overcome this drawback.

We used polyion complex method to prepare polymer nanosheets having ruthenium complexes $\left(\mathrm{Ru}(\mathrm{dpphen})_{3}{ }^{2+}\right)$. Spreading a mixed solution of poly( $N$-dodecylacrylamide-co-acrylic acid) with $\mathrm{Ru}(\text { dpphen })_{3}{ }^{2+}$ on a water surface, the luminescent polymer $(\mathrm{p}(\mathrm{DDA} / \mathrm{Ru}))$ nanosheets, in which $\mathrm{Ru}(\mathrm{dpphen})_{3}{ }^{2+}$ is uniformly distributed, were transferred onto the solid substrate using the LB technique. The concentration of $\mathrm{Ru}(\mathrm{dpphen})_{3}{ }^{2+}$ is adjustable by varying the contents of acrylic acid group and $\mathrm{Ru}(\text { dpphen })_{3}{ }^{2+}$. Figure 9 shows luminescence spectra of $\mathrm{p}(\mathrm{DDA} / \mathrm{Ru}$ ) nanosheets (four layers, excitation wavelength $=460 \mathrm{~nm}$ ) with or without a silver nanoparticle monolayer, as a function of oxygen concentration. In both cases, the luminescence intensity de-

(a)
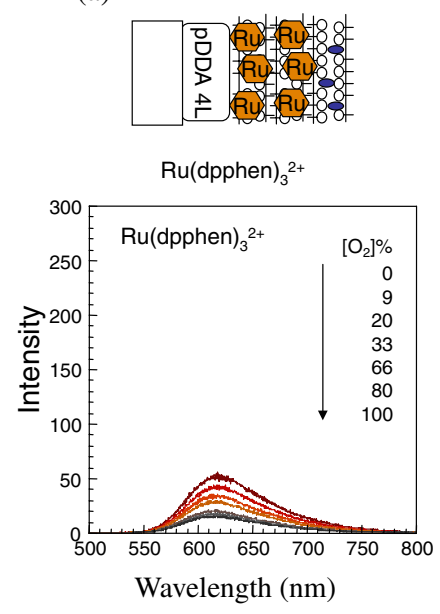

creases as the oxygen concentration increases. Assembling a silver nanoparticle $(80 \mathrm{~nm} \varphi)$ monolayer on the $\mathrm{p}(\mathrm{DDA} / \mathrm{Ru}$ ) nanosheets (Figure $9(\mathrm{~b})$ ), fourfold luminescence intensity enhancement was achieved in an argon atmosphere $\left(0 \% \mathrm{O}_{2}\right.$ concentration). Similar to the case of $\mathrm{Ru}(\mathrm{bpy})_{3}{ }^{2+}$ in a previous report, ${ }^{46 \mathrm{a}}$ the surface plasmon electromagnetic field generated around silver nanoparticles enhances the excitation efficiency of $\mathrm{Ru}(\mathrm{dpphen})_{3}{ }^{2+}$ incorporated in $\mathrm{p}(\mathrm{DDA} / \mathrm{Ru})$ nanosheets. Linear Stern-Volmer plots were obtained for both $\mathrm{p}(\mathrm{DDA} / \mathrm{Ru})$ nanosheets and hybrid polymer nanoassemblies, indicating the uniform microenvironment around $\mathrm{Ru}(\mathrm{dpphen})_{3}{ }^{2+}$ (Figure 10). The hybrid nanostructure shows lower sensitivity to oxygen than that of single $\mathrm{p}(\mathrm{DDA} / \mathrm{Ru})$ system. Figure 11 shows the average lifetime of $\mathrm{Ru}(\mathrm{dpphen})_{3}{ }^{2+}$ as a function of oxygen concentration. The third harmonic frequency $(355 \mathrm{~nm})$ of a pulsed $\mathrm{Nd}$ :YAG laser was used as an excitation beam and luminescence was detected using a streak camera. Semilogarithmic plots of the time-resolved luminescence decay curves showed a curved character, but they were fitted easily with a sum of two exponential terms. The average lifetime was determined as

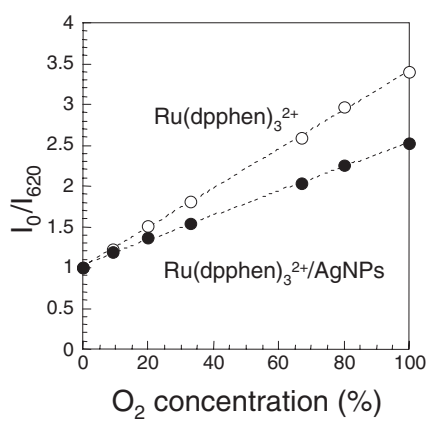

Figure 10. Stern-Volmer plots of single p(DDA/Ru) nanosheet system and hybrid nanoassembly.

(b)
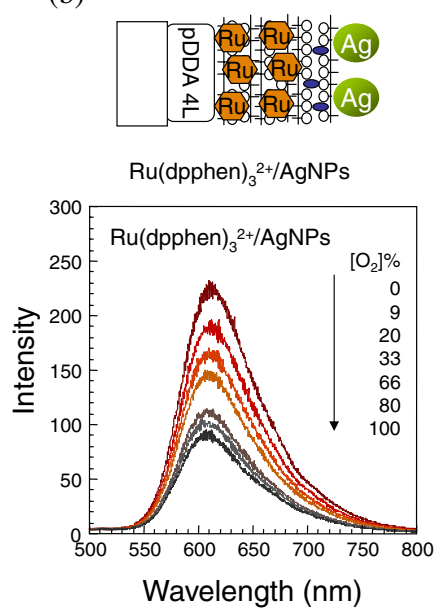

Figure 9. Luminescence spectra of (a) four-layer p(DDA/Ru) nanosheets and (b) four-layer p(DDA/Ru) nanosheets + AgNP monolayer. They were assembled on quartz slides. 


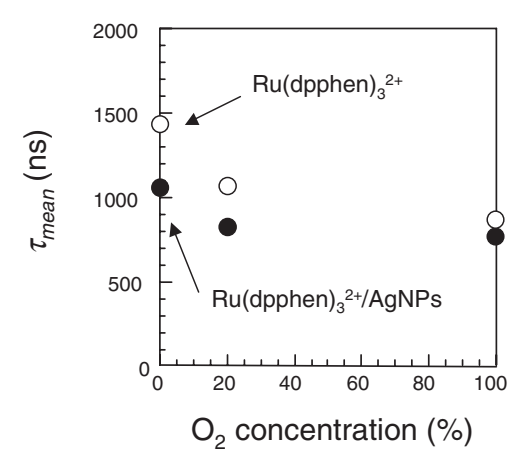

Figure 11. Plots of averaged luminescence lifetime as a function of oxygen concentration.

$$
\tau_{\text {mean }}=\frac{a_{1} \tau^{2}+a_{2} \tau^{2}}{a_{1} \tau+a_{2} \tau} .
$$

Interestingly, the average luminescence lifetime in argon atmosphere decreased when the $\mathrm{p}(\mathrm{DDA} / \mathrm{Ru})$ nanosheets were assembled with a silver nanoparticle monolayer. The enhanced luminescence intensity, the enhancement of the radiative rate, ${ }^{46 \mathrm{~b}}$ in addition to possible luminescence quenching by silver nanoparticle are plausible explanations of the decreased luminescence lifetime. Furthermore, the lifetime of the hybrid polymer nanoassemblies decreases as the oxygen concentration increases, but the sensitivity to oxygen becomes lower than that of $\mathrm{p}(\mathrm{DDA} / \mathrm{Ru})$ nanosheets alone. Consider two effects of sensitivity to oxygen: an increased radiative rate and luminescence quenching by silver nanoparticles. The luminescence quantum yield increases if the radiative rate enhancement is the case. Consequently, the decreased luminescence lifetime decreases the extent of luminescence quenching by oxygen. The luminescence quenching by silver nanoparticles will also lower the sensitivity to oxygen analogously. The silver nanoparticle monolayer has a broad extinction band at $620 \mathrm{~nm}$, overlapped with the luminescence band of $\mathrm{Ru}(\mathrm{dpphen})_{3}{ }^{2+}$. Thus, the silver nanoparticle serves as a quencher for excited $\mathrm{Ru}(\text { dpphen })_{3}{ }^{2+}$. The effect of separation distance on the change in luminescence intensity from $\mathrm{Ru}(\mathrm{bpy})_{3}{ }^{2+}$ implies that energy transfer quenching is a predominant factor of decreased luminescence intensity (data not shown). For practical purposes, it is noticeable that the sensitivity of $\mathrm{p}(\mathrm{DDA} / \mathrm{Ru})$ nanosheets in both $\mathrm{p}(\mathrm{DDA} / \mathrm{Ru})$ and its hybrid nanostructure for oxygen concentration is lower than that of $\mathrm{Ru}(\mathrm{dpphen})_{3}{ }^{2+}$ itself, probably because of the lesser accessibility of oxygen to $\mathrm{Ru}(\mathrm{dpphen})_{3}{ }^{2+}$ protected by long alkyl side chains. However, the response for oxygen concentration will be faster because of very ultrathin film thickness; in fact, the response was reversible and the film performance varied in a repeatable manner with oxygen concentration within $20 \mathrm{~s}$. The time response should be much faster because the change in oxygen concentration was a rate-determining process. Such luminescence enhancement has also been achieved in a metallo-porphyrin/silver nanoparticle system. ${ }^{47}$ We observed similar enhancement effects on metallo-porphyrin incorporated in polymer nanosheets. Details related to those observations will be reported in the near future.

\section{SECOND HARMONIC GENERATION FROM HYBRID POLYMER NANOASSEMBLIES}

Optical second harmonic generation $(\mathrm{SHG})$ requires noncentrosymmetric structures of nonlinear optically (NLO) active molecules; consequently, LB technique is of interest because it enables orientation control of NLO material at the molecular level. ${ }^{48-50}$ When LB films are fabricated in the vertical dipping method, the resultant LB films have two consecutive layers with either head-to-head or tail-to-tail type structures, thereby creating a centrosymmetric structure. The formation of that structure can be avoided by alternate deposition $^{51}$ of the NLO active molecular layer with a passive spacer molecular layer. We demonstrated a nanoscale combination of NLO active polymer nanosheet and a gold nanoparticle array and achieved SHG enhancement from hybrid polymer nanoassemblies to further improve NLO properties of LB films. ${ }^{52}$ Noble metal nanoparticles have large $\chi^{(3)}$ susceptibility values; ${ }^{53}$ therefore, nonlinear optical characteristics of nanoparticles have been investigated intensively, in particular, for third harmonic generation. As for the SHG efficiency of the nanoparticle, however, the $\chi^{(2)}$ values of noble metal nanoparticles are very small, which imparts modest NLO effects: $\chi^{(2)}=0$ for a perfectly spherical nanoparticle. The combination of an NLO active polymer nanosheet and a gold nanoparticle array facilitates effective surface plasmon coupling with SHG.

An amphiphilic NLO active copolymer (p(DDA/ DR)) was designed and synthesized through free radical copolymerization. The molecular weight and DR content were determined with GPC (polystyrene standard) and UV-Vis spectroscopy: $M_{\mathrm{n}}=2.80 \times$ $10^{3}, M_{\mathrm{w}} / M_{\mathrm{n}}=1.58,18 \mathrm{~mol} \%$ DR content (Figure 12). The pDDA was used as an inert layer to create a noncentrosymmetric layer structure. Alternate LB film deposition was carried out with an automatically controlled LB trough. A glass substrate was rendered hydrophobic using octyltrichlorosilane. The surface was precoated with four-layer pDDA nanosheets to avoid surface effects. We constructed hetero-structured polymer nanosheets by transferring $\mathrm{p}(\mathrm{DDA} / \mathrm{DR})$ (downstroke) and pDDA (upstroke) alternately at surface pressures and temperature of $30.0 \mathrm{mN} / \mathrm{m}$ for 


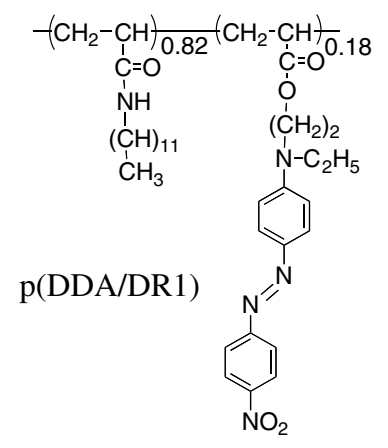

Without Au NPs

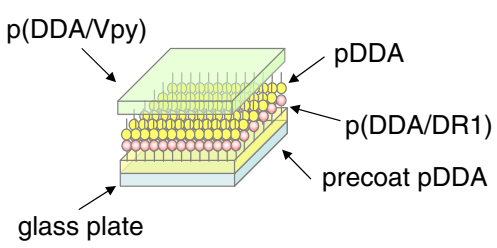

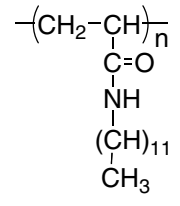

pDDA

With Au NPs

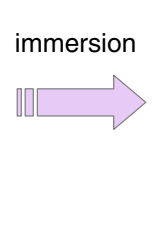

Au nanoparticle

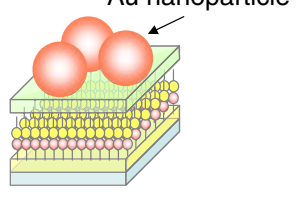

Figure 12. Schematic illustration of hybrid polymer nanoassemblies for SHG enhancement.

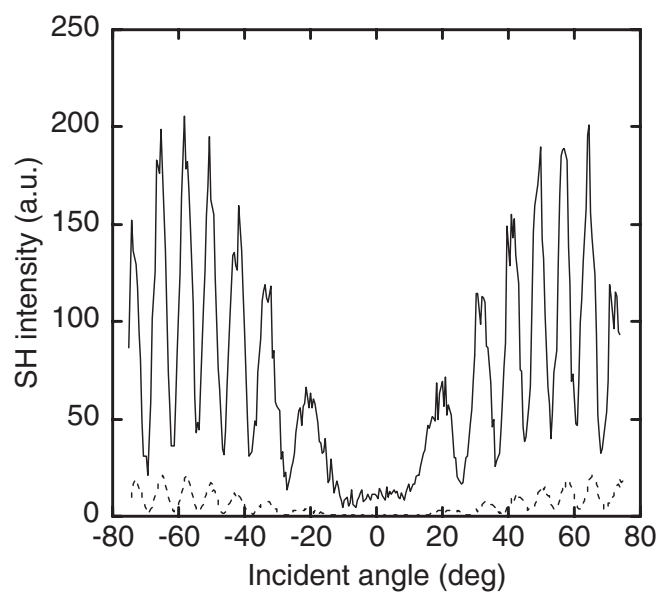

Figure 13. Second harmonic fringe patterns. Reprinted with permission from ref 52. Copyright 2006, American Institute of Physics.

pDDA, $15.0 \mathrm{mN} / \mathrm{m}$ for $\mathrm{p}(\mathrm{DDA} / \mathrm{DR})$, and $20.0^{\circ} \mathrm{C} .^{51}$ Two-layer cationic polymer nanosheets, $\mathrm{p}(\mathrm{DDA} /$ VPy) (Figure 2) at $35 \mathrm{mN} / \mathrm{m}$ and $15.0^{\circ} \mathrm{C}$, were deposited on the hetero-deposited polymer nanosheets. The substrate was immersed in a gold nanoparticle $(30 \mathrm{~nm}$ $\varphi$ ) aqueous solution.

Figure 13 shows the SH light interference pattern obtained from one bilayer film of $\mathrm{p}(\mathrm{DDA} / \mathrm{DR})$ and pDDA coated on both sides of the glass substrates (Figure 13, dashed line). The p-light component of fundamental frequency $(1064 \mathrm{~nm})$ from a nanosecond-pulsed Nd:YAG laser was incident on the sample and the p-component of the second harmonic light $(532 \mathrm{~nm})$ was detected by a photomultiplier as a function of the incident angle. Dependence of the SH light intensity on the incident angle shows a well-contrasted fringe pattern resulting from interference between the emitted SH light produced by the front and back layers. Interestingly, the SH light intensity is enhanced remarkably under gold nanoparticle arrays (Figure 13, solid line). Eight-fold SH light intensity enhancement was achieved. The clear SH light interference pattern also indicates that the gold nanoparticle arrays are immobilized uniformly on the heterostructured polymer nanosheets and thereby produce a nanoparticle monolayer, engendering no aggregate formation that would affect the light coherence and scattering.

We measured UV-Vis extinction spectra of hybrid polymer nanoassemblies as a function of immersion time in a gold nanoparticle aqueous solution. The number of gold nanoparticles immobilized on the polymer ultrathin film surface increased as the immersion time increased. Nearer gold nanoparticles generate a coupled surface plasmon electromagnetic field, thereby causing a red shift of the extinction band broadened to $1500 \mathrm{~nm}$. Figure 14 shows the absorbance at $1064 \mathrm{~nm}$ and the SH light intensity of hybrid polymer nanoassemblies with different immersion times. Interestingly, no remarkable SH light intensity enhancement was observed below $8 \mathrm{~h}$, and eight-fold enhancement was achieved at $12 \mathrm{~h}$ immersion time. The change in SH light intensity is closely coincident with that in optical extinction at $1064 \mathrm{~nm}$, although the extinction at $532 \mathrm{~nm}$ increases monotonically. As the immersion time surpasses $6 \mathrm{~h}$, the gold nanoparticles increase to the extent that they mutually interact electromagnetically. The electromagnetic field enhancement at $1064 \mathrm{~nm}$ is attributable to the dipole-like coupled surface plasmon mode generated between adjacent gold nanoparticles. The enhanced electromagnetic field at $1064 \mathrm{~nm}$, generated from coupled surface 

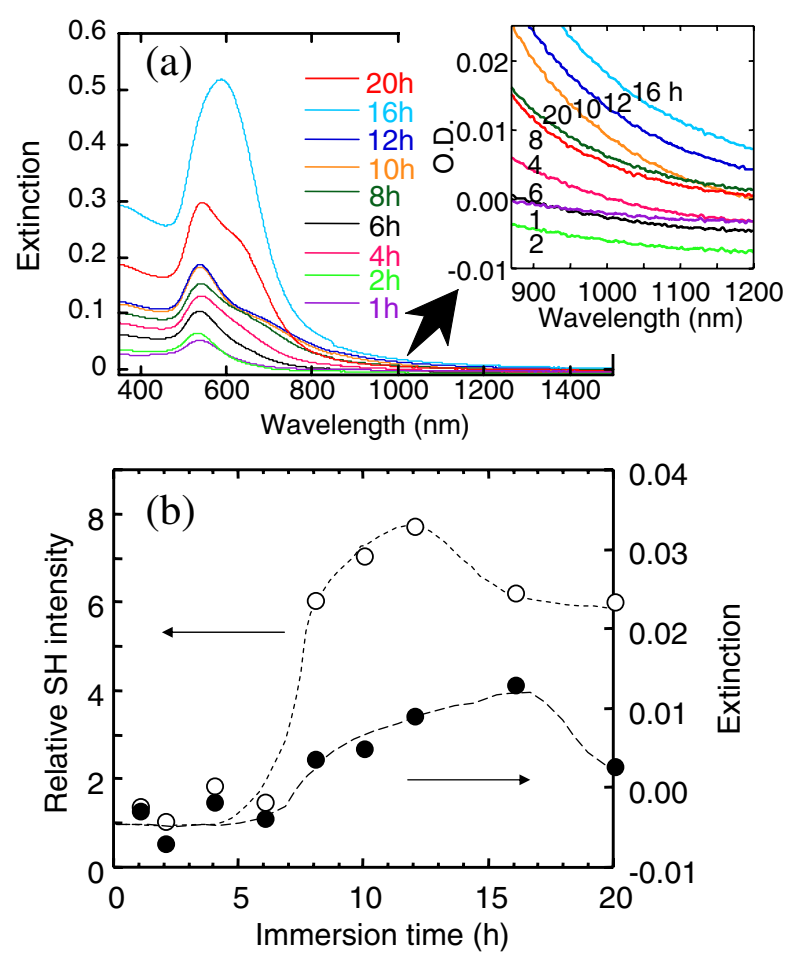

Figure 14. Changes in SH light intensity and absorbance at $1064 \mathrm{~nm}$ as a function of immersion time. Reprinted with permission from ref 52. Copyright 2006, American Institute of Physics.

plasmon resonance, excites nonlinear polarization of $\mathrm{DR}$, thus enhancing the SH light intensity at $532 \mathrm{~nm}$, which is a plausible explanation for the SH light intensity enhancement. It is noteworthy that SH light intensity enhancement was also obtained when the s-component of incident light impinged on the sample. These results strongly suggest that gold nanoparticle ordering for creating coupled surface plasmon electromagnetic field and hybridization of NLO active molecules with gold nanoparticle arrays at the nanometer scale are important for SH-light-intensity enhancement based on localized surface plasmon resonance.

Gold nanoparticles used in craftwork have shown beautiful colors, with their colors maintained exactly as they were several hundred years ago. Their optical properties were then exploited for coloration of glass, ceramics, china, and pottery. These optical properties arise from individual localized surface plasmons of gold nanoparticles; the various hues of the nanoparticles have been ascribed to various nanoparticle sizes, nanoparticle shape, stages of agglomeration, and overall composition. Recently, surface plasmon coupling, also known as particle-particle coupling, has received considerable attention. ${ }^{54-56}$ For widely separated nanoparticles, they behave independently. As that particle separation decreases, the dipolar response redshifts as described above, which also implies that color tuning as a function of interparticle spacing is possible with a well-defined nanostructure. Control

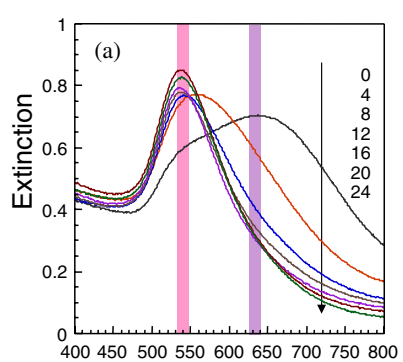

Wavelength $(\mathrm{nm})$

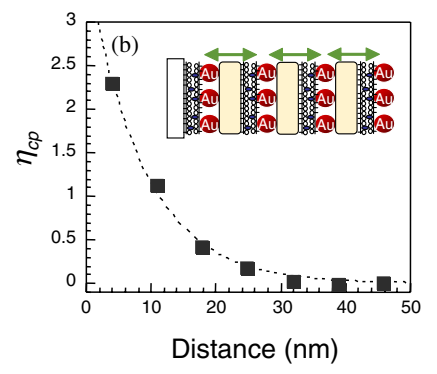

Figure 15. (a) UV-Vis extinction spectra of gold nanoparticle multilayers (after four deposition cycles) as a function of the number of pDDA spacer layers. The values show the numbers of pDDA spacer layers. (b) Plots of the surface plasmon coupling efficiency as a function of spacer distance between adjacent gold nanoparticle monolayers. The inset shows the layer structure.

of the surface plasmon coupling at the nanometer scale is achieved by varying the interlayer distance between gold nanoparticle monolayers with the number of inserting pDDA nanosheets. The gold nanoparticle appears scarlet in water, which is also the case for a gold nanoparticle monolayer immobilized on two-layer p(DDA/DADOO) nanosheets after $4 \mathrm{~h}$ immersion in an aqueous gold nanoparticle solution. The gold nanoparticle monolayer comprised uncoupled and isolated nanoparticles, which implies that no interparticle coupling occurred between adjacent gold nanoparticles in the monolayer because of its well-separated configuration. After repeating two-layer p(DDA/ DADOO) nanosheet deposition and gold nanoparticle adsorption with an $n$-layer $(n=0,4,8,12,16,20,24)$ pDDA spacer four times, UV-Vis extinction spectral measurement was implemented (Figure 15(a)). The extinction spectra in the extinction peaks at 538 and $637 \mathrm{~nm}$ are assigned respectively to isolated particle and interparticle surface plasmon coupling. It is noteworthy that the extinction peak for isolated gold nanoparticles redshifts because the environment moves from air to pDDA nanosheets. For $n=0$, no pDDA nanosheets were inserted between gold nanoparticle monolayers. Also, dipole-like surface plasmon coupling between two adjacent gold nanoparticle monolayers was the strongest, resulting in the red shift and broadening of the extinction band. The extinction band shape resembles that of gold nanoparticle in solution when the number of pDDA layers increases. 
Using the two optical extinction values at 538 and $637 \mathrm{~nm}$, the efficiency of surface plasmon coupling is estimated as

$$
\eta_{c p}=\frac{\left(A_{637} / A_{538}\right)_{n}-\left(A_{637} / A_{538}\right)_{24}}{\left(A_{637} / A_{538}\right)_{24}},
$$

where $\mathrm{A}_{538}$ and $\mathrm{A}_{637}$ represent optical extinction at 538 and $637 \mathrm{~nm}$. Figure 15(b) illustrates the distancedependent behavior for interlayer surface plasmon coupling: the $\eta_{\mathrm{cp}}$ value decreases exponentially as the distance between gold nanoparticle monolayers increases. Assuming that the monolayer thicknesses of $\mathrm{p}(\mathrm{DDA} / \mathrm{DADOO})$ and $\mathrm{pDDA}$ are 2.0 and $1.7 \mathrm{~nm}$, the separation distance between gold nanoparticle monolayers is determined. The experimental data were fitted with a single exponential function (solid line, Figure 15(b)). Consequently the decay distance $\left(d_{1 / e}\right)$, of which the magnitude falls to $1 / e$, was determined as $d_{1 / e}=8.7 \mathrm{~nm}$, indicating that effective interlayer surface plasmon coupling occurs within the spacer separation distance of $8.7 \mathrm{~nm}$ for a $30 \mathrm{~nm} \phi$ gold nanoparticle. Although quantitative analyses involving surface density of gold nanoparticle in the monolayer are necessary to yield more detailed information, this approach provides a basis for understanding important interactions that occur in hybrid polymer nanoassemblies.

The photographs in Figure 16 show a clear progression in coloration. For more numerous pDDA spacer layers, the colors progressively became violet-purple, purple, purple-red, and finally ruby-red. The colors intensified with more layers. The resulting films showed metallic colors when the gold nanoparticle was immobilized for $20 \mathrm{~h}$ and assembled three times with no pDDA spacer layers. The gold nanoparticles were optically coupled through intra-layer and inter-layer dipolar interactions, but no electronic coupling exists.

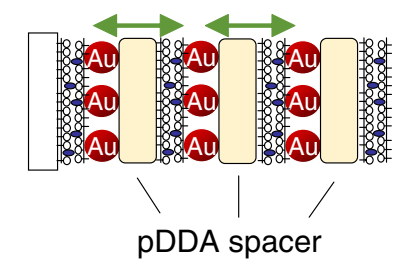

(a)

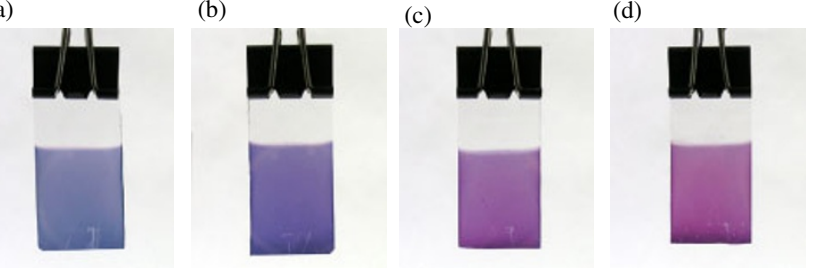

Figure 16. Photographs of gold nanoparticle multilayers with different numbers of pDDA layers after their respective third deposition cycles. The numbers of pDDA spacer layers: (a) 0 , (b) 4 , (c) 8, and (d) 24 layers.
All these findings reflect well-defined 3D nanostructures of ultrathin polymer nanosheets and metal nanoparticle arrays, which enable control of surface plasmon coupling at the nanometer scale.

\section{CONCLUSION}

We described fascinating aspects of hybrid polymer nanoassemblies consisting of polymer nanosheets and noble metal nanoparticle arrays: preparation of freestanding ultrathin films, luminescence enhancement for luminescence sensor application, and enhancing SH light intensity under surface plasmon coupling between two gold nanoparticles. The advantage of this approach lies in precise, nanometer-scale positioning of noble metal nanoparticles and functional molecules along with the film surface normal. By varying the functional group's molar contents and immersion time in an aqueous metal nanoparticle solution, reproducible and precise control of metal nanoparticle immobilization was achieved. This approach provides a suitable configuration for a deep understanding of the relationship between the materials' spectroscopic properties and localized surface plasmon generated from metal nanoparticles: a well separated metal nanoparticle monolayer was stacked on the functional polymer nanosheets. This approach also enables fruitful characterization of the interaction between metal nanoparticles and functional molecules in hybrid polymer nanoassemblies. Because of its strong extinction (absorption and scattering) in the visible light region, it is possible to realize a free-standing film even though its thickness corresponds to the nanoparticle diameter. The resultant free-standing film is robust and flexible: it is highly anticipated as a next-generation nanomaterial. To use localized surface plasmons effectively, the importance of the hybrid nanoassembled structure with metal nanoparticles ( $\mathrm{Au}$ and $\mathrm{Ag}$ ) and photo/electro functional molecules at nanometer precision should be emphasized because localized surface plasmons are confined to the metal surface. An understanding of the relationship between material properties and optical/spectroscopic properties of functional molecules will open up new avenues for future nanodevice applications. For example, characterization and functionalization of free-standing films remains as an important issue to be clarified. In addition, the strong extinction band from metal nanoparticles implies that the metal nanoparticle serves as an effective quencher when the nanoparticle is coupled with a suitable luminophore. In particular, this tendency is observed for smaller metal nanoparticles. In this sense, detailed investigation of luminescence or SHG properties of hybrid polymer nanoassemblies will provide a deep understanding of localized surface plas- 
mon coupling with functional molecules. Some of these issues are being addressed by work in progress. Their results will be published in future reports.

Acknowledgment. The work was supported by Grants-in Aid for Scientific Research ((S) No. 17105006), Young Scientists ((A), No. 18685027), and Priority Areas (No. 417, No. 17029006).

\section{REFERENCES}

1. G. Decher and J. Schienoff, in "Multilayer Thin Films: Sequential Assembly of Nanocomposite Materials," Wiley-VCH, Weinheim, 2002.

2. R. C. Advincula, W. J. Brittain, K. C. Caster, and J. Rühe, in "Polymer Brushes," Wiley-VCH, Weinheim, 2004.

3. T. Miyashita, Prog. Polym. Sci., 18, 263 (1993).

4. a) G. Wegner, Macromol. Chem. Phys., 204, 347 (2003). b) V. V. Tsukruk, Prog. Polym. Sci., 22, 247 (1997).

5. S. Ito, M. Mabuchi, N. Sato, and H. Aoki, Bull. Chem. Soc. Jpn., 78, 371 (2005).

6. A. Ulman, in "An Introduction to Ultrathin Organic Films from Langmuir-Blodgett to Self-Assembly," Academic Press Inc., New York, 1991.

7. M. C. Petty, in "Langmuir-Blodgett Films," Cambridge University Press, Cambridge, 1996.

8. T. Miyashita, J. Matsui, and M. Mitsuishi, in "Functional Nanomaterials," K. E. Geckeler and E. Rosenberg, Ed., American Scientific Publishers, Valencia, 2006, p 361.

9. M. Mitsuishi, J. Matsui, and T. Miyashita, Polym. J., 38, 877 (2006).

10. A. Aoki, Y. Abe, and T. Miyashita, Langmuir, 15, 1463 (1999).

11. J. Chen, M. Mitsuishi, A. Aoki, and T. Miyashita, Chem. Commun., 2856 (2002).

12. J. Matsui, M. Mitsuishi, A. Aoki, and T. Miyashita, Angew. Chem., Int. Ed., 42, 2272 (2003).

13. J. Matsui, M. Mitsuishi, A. Aoki, and T. Miyashita, J. Am. Chem. Soc., 126, 3708 (2004).

14. Y. Kado, M. Mitsuishi, and T. Miyashita, Adv. Mater., 17, 1857 (2005).

15. T. Li, J. Chen, M. Mitsuishi, and T. Miyashita, J. Mater. Chem., 13, 1565 (2003).

16. A. Aoki and T. Miyashita, Adv. Mater., 9, 361 (1997).

17. T. Mikayama, T. Suzuki, J. Matsui, and T. Miyashita, Polym. J., 37, 854 (2005).

18. M. Mitsuishi, T. Li, and T. Miyashita, Langmuir, 18, 10035 (2002)

19. N. Fukuda, M. Mitsuishi, A. Aoki, and T. Miyashita, J. Phys. Chem. B, 106, 7048 (2002).

20. a) K. G. Grabar, R. G. Freeman, M. B. Hommer, and M. J. Natan, Anal. Chem., 67, 735 (1995).

b) A. N. Shipway, E. Katz, and I. Willner, Chem. Phys. Chem., 1, 18 (2000).

21. M. C. Daniel and D. Astruc, Chem. Rev., 104, 293 (2004).

22. A. Moores and F. Goettmann, New J. Chem., 30, 1121 (2006).

23. E. Hutter and J. H. Fendler, Adv. Mater., 16, 1685 (2004).
24. a) K. Ohno, K. Koh, Y. Tsujii, and T. Fukuda, Angew. Chem., Int. Ed., 42, 2751 (2003).

b) C. Jiang, S. Markutsya, and V. V. Tsukruk, Langmuir, 20, 882 (2004).

25. a) H. Tanaka, M. Mitsuishi, and T. Miyashita, Langmuir, 19, 3103 (2003).

b) M. Mitsuishi, H. Tanaka, and T. Miyashita, Trans. Mater. Res. Soc. Jpn., 30, 639 (2005).

26. M. J. Hostetler, J. E. Wingate, C. J. Zhong, J. E. Harris, R. W. Vashet, M. R. Clark, J. D. Londono, S. J. Green, J. J. Stokes, G. D. Wignall, G. L. Glish, M. D. Porter, N. D. Evans, and R. W. Murray, Langmuir, 14, 17 (1998).

27. C. Jiang and V. V. Tsukruk, Adv. Mater., 18, 829 (2006).

28. S. Markutsya, C. Jiang, Y. Pikus, and V. V. Tsukruk, $A d v$. Funct. Mater., 15, 771 (2005).

29. A. A. Mamedov and N. A. Kotov, Langmuir, 16, 5530 (2000).

30. F. Mallwitz and W. A. Goedel, Angew. Chem., Int. Ed., 40, 2645 (2001).

31. R. Vendamme, S. Onoue, A. Nakao, and T. Kunitake, Nat. Mater., 5, 494 (2006).

32. A. A. Mamedov, N. A. Kotov, M. Prato, D. M. Guldi, J. P. Wicksted, and A. Hirsch, Nat. Mater., 1, 190 (2002).

33. J. Jin, J. Huang, and I. Ichinose, Angew. Chem., Int. Ed., 44, 4532 (2005).

34. C. Jiang, S. Markutsya, H. Shulha, and V. V. Tsukruk, Adv. Mater., 17, 1669 (2005).

35. H. Endo, Y. Kado, M. Mitsuishi, and T. Miyashita, Macromolecules, 39, 5559 (2006).

36. T. Ung, L. M. Liz-Marzán, and P. Mulvaney, J. Phys. Chem. $B, \mathbf{1 0 5}, 3441$ (2001).

37. C. F. Bohren, D. R. Huffman, in "Absorption and Scattering of Light by Small Particles," Wiley-VCH, Weinheim, 2004.

38. U. Kreibig and M. Vollmer, in "Optical Properties of Metal Clusters," Springer, Heidelberg, 1995.

39. T. R. Jensen, M. D. Malinsky, C. L. Haynes, and R. P. Van Duyne, J. Phys. Chem. B, 104, 10549 (2000).

40. a) A. J. Haes and R. P. Van Duyne, Anal. Bioanal. Chem., 379, 920 (2004).

b) S. Link, Z. L. Wang, and M. A. El-Sayed, J. Phys. Chem. B, 103, 3529 (1999).

41. O. S. Wolfbeis, in "Fiber Optic Chemical Sensors and Biosensors," O. S. Wolfbeis, Ed., CRC Press, London, 1993.

42. L. Sacksteder, J. N. Demas, and B. A. DeGraff, Anal. Chem., 65, 3480 (1993).

43. B. W.-K. Chu and V. W.-W. Yam, Langmuir, 22, 7437 (2006).

44. B.-H. Han, I. Manners, and M. A. Winnik, Chem. Mater., 17, 3160 (2005).

45. T. Miyashita, J. Chen, M. Yuasa, and M. Mitsuishi, Polym. J., 31, 1121 (1999).

46. a) H. Tanaka, M. Mitsuishi, and T. Miyashita, Chem. Lett., 34, 1246 (2005).

b) J. R. Lakowicz, Y. Shen, S. D'Auria, J. Malicka, J. Fang, Z. Gryczynski, and I. Gryczynski, Anal. Biochem., 301, 261 (2002).

47. S. Pan and L. J. Rothberg, J. Am. Chem. Soc., 127, 6087 (2005).

48. G. J. Ashwell, J. Mater. Chem., 9, 1991 (1999). 
49. K. Kajikawa, T. Yamaguchi, T. Anzai, H. Takezoe, A. Fukuda, S. Okada, H. Matsuda, H. Nakanishi, T. Abe, and H. Ito, Langmuir, 8, 2764 (1992).

50. W. M. K. P. Wijekoon, S. K. Wijaya, J. D. Bhawalkar, P. N. Prasad, T. L. Penner, N. J. Armstrong, M. C. Ezenyilimba, and D. J. Williams, J. Am. Chem. Soc., 118, 4480 (1996).

51. a) F. Grunfeld, Rev. Sci. Instrum., 64, 548 (1993).

b) M. Ishifuji, M. Mitsuishi, and T. Miyashita, Nonlinear Opt. Quant. Opt., 34, 111 (2005).

52. M. Ishifuji, M. Mitsuishi, and T. Miyashita, Appl. Phys.
Lett., 89, 011903 (2006).

53. F. Hache, D. Ricard, and C. Flytzanis, J. Opt. Chem. Soc. Am. B, 3, 1647 (1986).

54. I. Romero, J. Aizpurua, G. W. Bryant, and F. J. García de Abajo, Opt. Exp., 14, 9988 (2006).

55. K.-H. Su, Q.-H. Wei, X. Zhang, J. J. Mock, D. R. Smith, and S. Schultz, Nano Lett., 3, 1087 (2003).

56. W. Rechberger, A. Hohenau, A. Leitner, J. R. Krenn, B. Lamprecht, and F. R. Aussenegg, Opt. Commun., 220, 137 (2003).
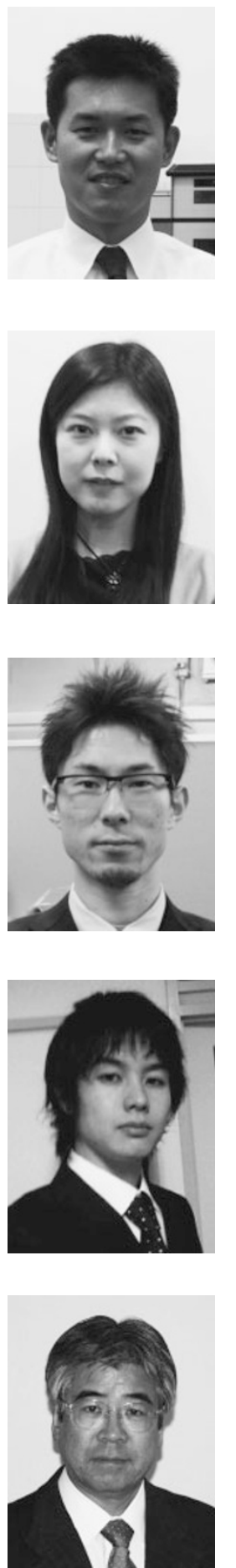

Prof. Tokuji Miyashita (Vice President, the Society of Polymer Science, Japan (SPSJ) in 2006 and 2007) was born in Yamanashi, Japan in 1948. He received his Bachelor's (1971), Master's (1973) and Doctorate (1976) degrees from Tohoku University's Faculty of Engineering. He was promoted to Professor in 1993 at the Department of Engineering, Tohoku University. He is currently a Deputy Director at the Institute of Multidisciplinary Research for Advanced Materials, Tohoku University. He has received several awards including the Award of the Society of Polymer Science, Japan for 1996 and the SPACC-CSJ Award for 2003. His research interests are materials science and technology for soft nano-system architectures.

Masaya Mitsuishi was born in Osaka, Japan in 1968. He received his Ph.D. from Kyoto University in 1997 under the direction of Prof. Masahide Yamamoto. In that year, he joined the group of Prof. Tokuji Miyashita at the Institute of Chemical Reaction Science, Tohoku University, as a Postdoctoral Research Associate. He was promoted to Associate Professor in 2004. He received the Award for the Encouragement of Research in Polymer Science of the Society of Polymer Science, Japan (2002) and The SPSJ Hitachi Chemical Award (2006). His current research interests include photofunctional polymers, polymer nanoassemblies, and photofunctionality based on evanescent wave optics.

Miki Ishifuji was born in Iwate, Japan in 1980. She received her Bachelor's degree in 2004 from Tokyo University of Science and Master's degree in 2006 from Tohoku University. Now she is working at Tohoku University toward her Ph.D. under the guidance of Prof. Tokuji Miyashita and Assoc. Prof. Masaya Mitsuishi. Her research concerns enhanced nonlinear optical phenomena using surface plasmon resonance in polymer hybrid nanoassemblies.

Hiroshi Endo was born in Yamagata, Japan in 1978. He received his Bachelor's degree (2001) and Master's degree (2004) in chemical and biochemical engineering from Toyama University under the supervision of Prof. Hiromi Kitano. In 2004, he joined Prof. Miyashita's group at Tohoku University. He is now focusing on free-standing polymer films using Langmuir-Blodgett method under the supervision of Prof. Tokuji Miyashita and Assoc. Prof. Masaya Mitsuishi. His current research interests include polymer thin films, organic-inorganic nanocomposites, and supramolecular materials.

Hiroyuki Tanaka was born in Chiba, Japan in 1979. He received his Bachelor's degree (2002) and Master's degree (2004) from Tohoku University under the direction of Prof. Tokuji Miyashita. In 2004, he proceeded to the Ph.D. course of engineering at Tohoku University. He is now studying metal nanoparticle-polymer hybrid nanoassemblies using Langmuir-Blodgett method under the direction of Prof. Tokuji Miyashita and Assoc. Prof. Masaya Mitsuishi. His current research interests include photofunctional polymers, nanoparticle-polymer hybrid materials, and photofunctionality based on localized surface plasmons. 